\title{
Téoros
}

Revue de recherche en tourisme

\section{Le parc du Mont-Tremblant : 100 ans de nature!}

\section{André B. Lemay, Pierre Dupuy, Brigitte Dubé et Johanne Forest}

Volume 14, numéro 1, printemps 1995

Les parcs : des réseaux en mutation

URI : https://id.erudit.org/iderudit/1077047ar

DOI : https://doi.org/10.7202/1077047ar

Aller au sommaire du numéro

Éditeur(s)

Université du Québec à Montréal

ISSN

0712-8657 (imprimé)

1923-2705 (numérique)

Découvrir la revue

Citer cet article

Lemay, A. B., Dupuy, P., Dubé, B. \& Forest, J. (1995). Le parc du

Mont-Tremblant : 100 ans de nature! Téoros, 14(1), 3-7.

https://doi.org/10.7202/1077047ar 


\title{
Le parc du Mont-Tremblant : 100 ans de nature!
}

\author{
André B. Lemay, Pierre Dupuy, Brigitte Dubé et Johanne Forest"
}

Il y a de cela bien longtemps, les Amérindiens, premiers occupants de ce coin de pays, donnaient à la haute montagne qui domine le paysage du parc du MontTremblant, le nom de Manitonga Soutana, c'est-à-dire kmontagne du Diable ou montagne de l'Esprit». Une légende prétendait que la montagne émettait des bruits sourds, pareils à des grondements, quand l'homme venait troubler la tranquillité des lieux.

Aujourd'hui, le manitou veille toujours sur cet immense territoire qui fut, il y a 100 ans, le premier au Québec à porter le nom de parc. Il rappelle au visiteur que cette terre a été protégée pour lui et pour ses enfants, qu'il en a non seulement hérité de son père, mais qu'il l'emprunte à sa progéniture.

S'aventurer dans ce «pays-d'en-hauts, c'est sillonner un territoire en constante évolution, au rythme d'une société qui valorise de plus en plus son patrimoine naturel; c'est découvrir un parc qui entreprend son deuxième siècle d'existence en réaffirmant l'importance de sa mission de conservation, d'éducation et de récréation.

\section{Le premier parc au Québec}

Le vaste territoire aujourd'hui occupé par le parc du Mont-Tremblant était autrefois peu fréquenté; seuls quelques chasseurs, trappeurs, pêcheurs et explorateurs s'y aventuraient. Son relief accidenté, ses forêts denses, ses terres pauvres et son accès difficile rendaient ce pays plutôt hostile à l'établissement de colons. Par conséquent, le vaste mouvement de colonisation des Laurentides, mené par le célèbre curé Labelle et qui connait son apogée vers

\footnotetext{
Monsieur Andre B. Lemay est directeur par interim du parc du Mont-Tremblant. Monsieur Pierre Dupuy en est le responsable de la mise en valeur du milieu et de la protection. Mesdames Bngitte Dube et Johanne Forest sont respectivement agente d'information et chet du Service des communications et du marketing a la Direction récionale - Faune ì Montréal.
}

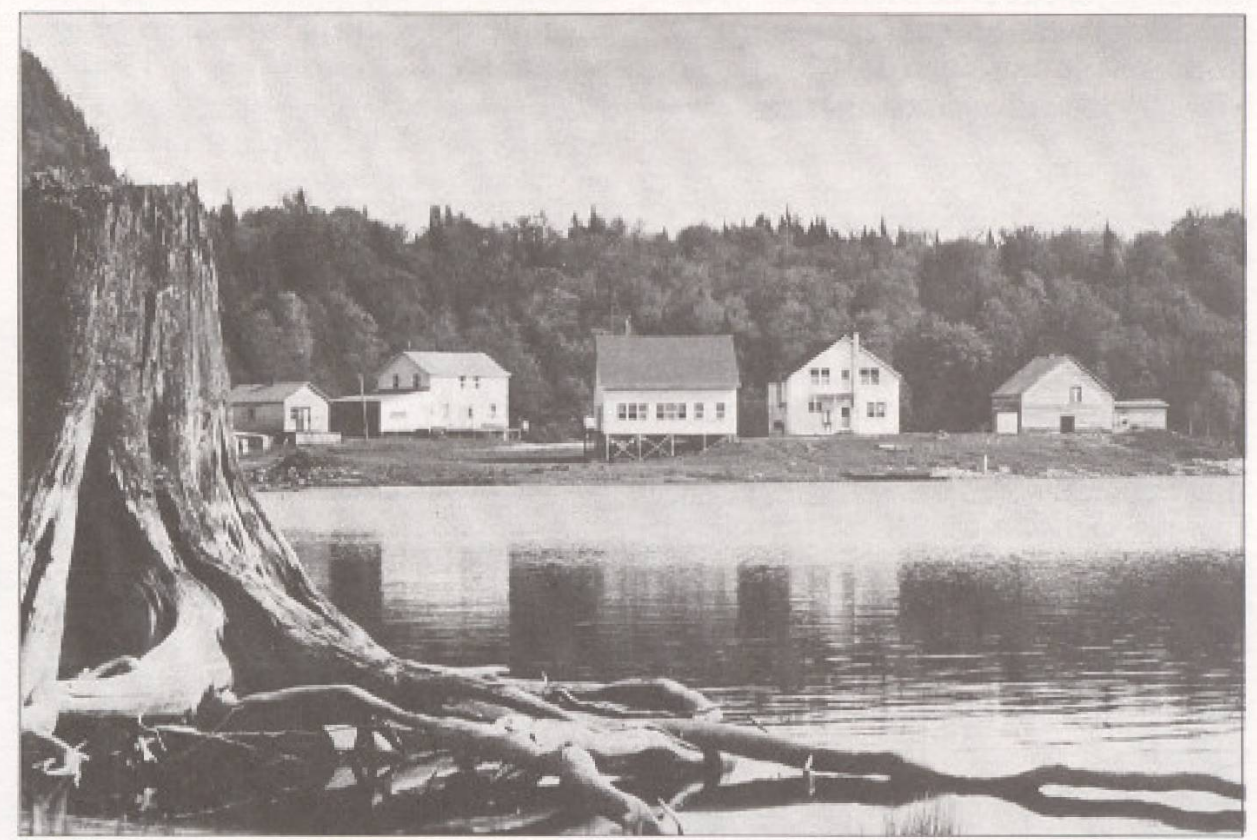

La Stution hiologique du Mont-Tremblant (Photo: parc du Mont-Tremblant)

1880 , n'atteindra pas ce territoire. Son milieu physique détermine donc, trì̀s tôt, si vocation forestière. Depuis le milieu du 19" sjècle, l'exploitation du bois constitue sa principale activité économique. Parallèlement, de la chasse et de la péche ont cours sur ses terres; des clubs privés s'y installent dès le début du $20^{c}$ siècle.

Au printemps de 1894, un médecin montréalais, le docteur Camille Laviolette, demande au gouvernement du Québec la concession de terres sur la Montagne Tremblante, dans le dessein d'y ouvrir un sanatorium. A son avis, la montagne offre les conditions climatiques idéales pour guếrit les maladies pulmonaires. Les autorités politiques acceptent de réserver l'étendue de forêt nécessaire pour établir le futur hôpital. Elles accordent done un statut de réserve forestière à la majeure partiedu massif, soit une super ficie de 59,7 $\mathrm{km}^{2}$. Cette décision doit toutefois obtenir la sanction de la législature, ce qui sera fait le 12 janvier 1895 , avecl'adoption d'une loi établissint formellement le eparc de la Montagne Tremblantes. Il faut préciser toutcfois qu'aucune disposition dans la loi établissant le parc de la Montagne Tremblante, ne touchait alors la protection des ressources naturelles. La coupede bois peut donc se poursuivre selon le principe des permis de coupe ainsi que le prélèvement de la ressource faunique par la clientèle des clubs privés.

Quant au projet du docteur Laviolette, il connaitra, par la suite, des difficultés et ne verra finalement pas le jour sur les terrains désignés. D'une façon inattendue, il aura cependant donné le coup d'envoi à la création du premier parc québécois. D'autres suivront: celui des Laurentides, la mêmeannée, etplustard ceuxdela Gaspésie puis du Mont-Orford. L'établissement de pares cesse jusque dans les années 70 , alors qu'on décide de poursuivre l'expansion du réseau. Aujourd'hui, on en compte dixsept et deux sont en voie de création.

Lors de sa naissance en 1895 , le parc de la Montagne Tremblante ne s'étendait que sur une soixantaine de kilomètres carrés. C'est en 1897 qu'on retrouve pour la première fois dans les textes législatifs, l'intention d'agrandir substantiellement sa 
superficie. Des clubs privés et des compagnies de bois, qui possédaient des droits dans le territoire pressenti pour l'agrandissement, s'opposent cependant à ce projet. En 1925 pourtant, legouvernement ajoute officiellement au parcun territoirede 3108 $\mathrm{km}^{2}$, issu principalement des cantons des comtés de Joliette et de Montcalm. C'est également à cette date que s'ajoutent à la loi des notions de protection de la faune et de la flore, qui en étaient jusqu'alors absentes.

\section{Une industrie touristique en ascension}

A cette époque, les Laurentides deviennent un rendez-vous de choix pour les touristes de Montréal et d'ailleurs, attirés par ses panoramas incomparables, ses lacs limpides et l'air pur de ses montagnes. Pour la population, l'industrie touristique grandissante apparaît comme une porte ouverte à la prospérité dans une région où l'économie connaît des difficultés.

Quantau parcdela Montagne Tremblante, il possède le potentiel naturel pour contribuer largement à l'essor touristique, mais il n'est toujours, à cette date, qu'une réserve forestière. Avec le développement de l'industrie des pâtes et papiers, l'exploitation de la matière ligneuse s'y poursuit alors sur une plus grande échelle.

L'implantation du ski dans les Laurentides vient jouer un rôle déterminant pour l'industrie touristique. On ne saurait parler de développement de cette discipline sportive sans souligner l'apport de Hermann Smith Johannsen. On raconte que c'est son agilité sur skis qui luivalut un surnom, celui de «Jackrabbio». Ce norvégien d'origine, passionné de la nature, sillonne à skis les forêts de la région depuis 1925. Il affirmait que les touristes seraient plus nombreux si on leur offrait un bon réseau de pistes, projer auquel il se consacte avec énergie. Déjà autour de 1928 , on assiste à l'aménagementdevéritables pistesdansla région. A partir de 1930, un fort engouement pour la pratique du ski, combiné à un réseau routier amélioré depuis la multiplication des automobiles, contribuent à une croissance extraordinaire du tourisme dans la région, désormais fréquentée en toutes saisons.

Avec l'arrivée du remonte-pente, puis du télésiège, le ski alpin supplante de plus en plus le ski de fond et les centres de ski alpin se multiplient. La région de Saint-JoviteMont-Tremblant devient l'une des plus populaires auprès des skieurs. En 1938, un Américain fortuné, Joe Ryan, emballé par le site, décide de faire du massif une station de sports d'une envergure sans précédent qu'il nommera Mont-Tremblant Lodge. Répondant aux pressions des citoyens des environs, qui voyaient dans ce projet une promesse d'essor économique, l'Assemblée législativedu Québec modifie, en 1939, la Loi du parc de la Montagne Tremblante en y ajoutant les notions de parc public et de lieu de délassement, favorisant ainsi le développement de la station de ski.

\section{Le parc devient accessible au public}

Au cours des année 1950, le processus d'industrialisation et d'urbanisation obligeait les amateurs d'activités de plein air à chercher les espaces verts toujours plus loindes villes, Leschambres de commerce, les conseils municipaux des environs ainsi que plusieurs organismes et citoyens font appel aux autorités provinciales pour l'ouverture d'un véritable parc destiné à des fins récréatives dans les Laurentides. Au printemps 1958, les journaux parlent de la possibilitéd'aménagement du parc. Cette même annéc, le gouvernement consent des fonds pour entreprendre les premiers travaux afin de le rendre accessible aux vacanciers.

Depuis 1949, le parc de la Montagne Tremblante est déjà, par ailleurs, le site d'une station biologique mise sur pied par l'Office de biologie du Québec. De 1949à 1962 , des biologistes, des chimistes, des physiciens er d'autres scientifiques y effectuent des travaux qui constituent une étape marquante dans l'avancement des connaissances en gestion de la faune aquatique au Québec.

Ce sont les dirigeants de cette station qui seront les instigateurs, à l'été 1958 , du premier camping public, au lac Chat. L'initiative connaît un succès immédiat; dès la première année de fréquentation, 10000 touristes viennent prendre un bain de nature dans le parc. Ce sera le point de départd'unesérie d'aménagementsd'ahord concentrés dans la vallée de la Diable, puis au nord de Saint-Donat, visunt à satisfaire les besoins des visiteurs dont le nombre s'accroit rapidement. En 1961, l'appella- tion même du parc change; on le nomme dorénavant «parc du Mont-Tremblants.

\section{Le virage vert}

L'année 1977 marque un tournant dans l'histoire des parcs au Québec avec l'adoptonde la Loisur les parcs. Celle-ciintroduit des dispositions d'une importance majeure qui permettront au réseau des parcs québécois de répondre à des critères reconnus internationalement. Parmi ceuxci, l'interdiction de la pratique de la chasse et de toute exploitation commerciale des ressources forestières, minières et énergétiques.

À la suite de l'adoption de cette loi, le parc du Mont-Tremblant, acquière ses lettres de noblesse et devient, le 31 mars 1981, un territoire orienté vers la conservation de ses ressources naturelles, mais également destiné à l'éducation et à la récréation. Ainsi ennoblie, sa mission s'apparente davantageà la notion de parc telle que définie par l'Union internationale pour la conservation de la nature (UICN).

La superficie du parc est alors ramenée à $1248 \mathrm{~km}^{2}$, soit l'espace nécessaire pour répondre à sa nouvelle vocation. Dans ses nouvelles limites, le parc perd sa partie nord auprofitdela nouvelle réserve RougeMatawin, mais il s'enrichit de la presque totalité de l'ancienne réserve de Joliette. Il vient ainsi chevaucher la région touristique des Laurentides et celle de Lanaudière.

De plus, à la demande d'un nombre important de participants aux audiences publiques de 1979 , le législateur consent à créer, dans les limites du parc, une zone d'affectation différée(ZAD) de $242 \mathrm{~km}^{2}$, où la coupe forestière sera autorisée pour une période de 10 ans. Comme prévu, cette portion de territoiresera réintégréedansle parc en 1990 , portant sa superficie à $1490 \mathrm{~km}^{2}$.

Bénéficiant d'un cadre législatif approprié depuis 1981, le parc du Mont-Tremblant est dorénavant outillé pour amorcer un virage vert. Ce passage d'un statut de réserve, où l'exploitation des ressources est permise, à celui de parc, où leur protection est assurée, se fera progressivement. Dans la poursuite de ses mandats de conservation, d'éducation et de récréation, le parc devra composer avec l'héritage du passé. 


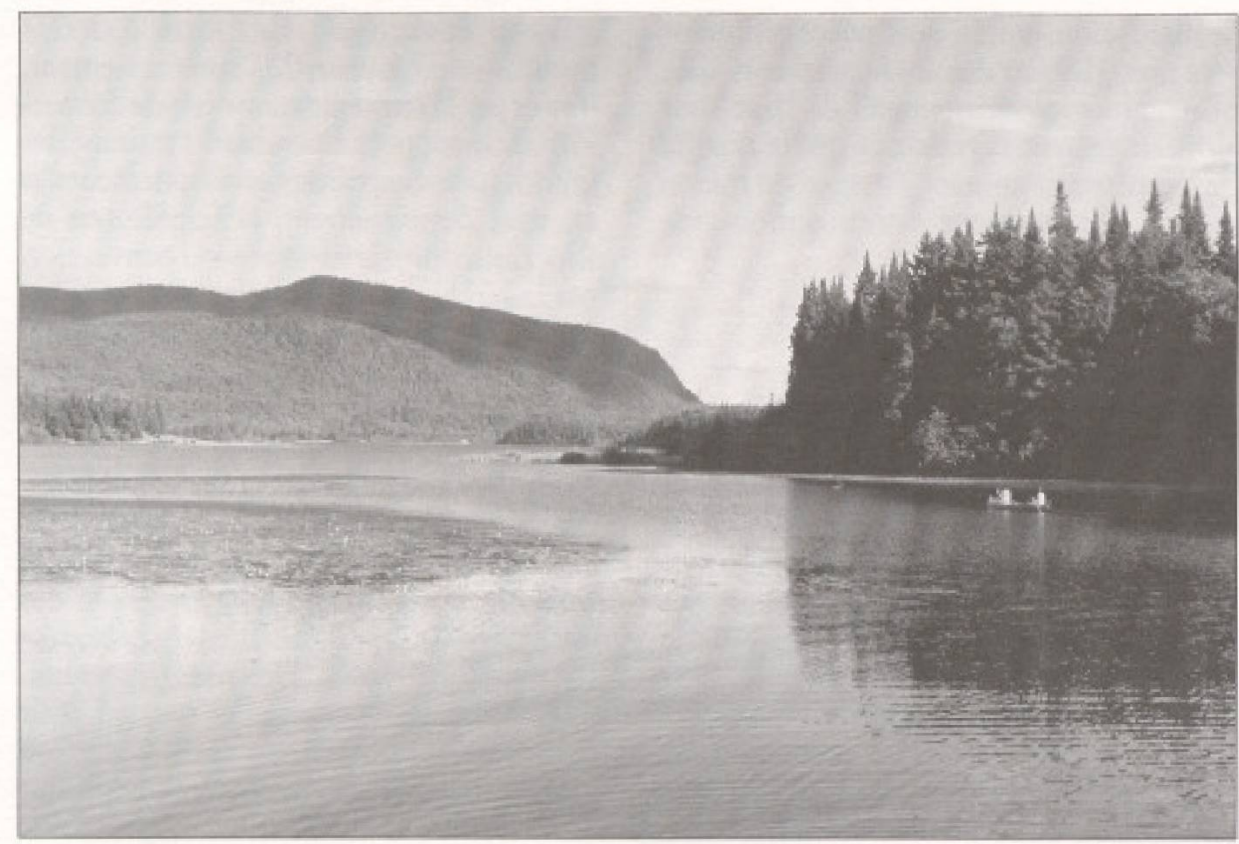

En canot, à la découverte du milieu naturel du parc du Mont Tremblant.

Photo: Sylvain Majeau / ministìre de l'Environnement et de lat Faune'

\section{Un paysage marqué par l'histoire}

Les montagnes, les rivières et les lacs du parc témoignent encore du labeur des défricheurs et des bûcherons du début du siècle. Principale activité ćconomique de survie dans l'arrière-pays, l'exploitation forestière a considérablement marqué le territoire. Au cours des cinquante dernières années, des formes diverses d'exploitation ont touché chaque kilomètre carré du parc. Tantôt intensive, tantôt plus restreinte, au grédes méthodes d'intervention forestière et des progrès technologiques, le passage de l'homme se reflète encore aujourd'hui sur le milieu.

Le résenu routier du parc, constituéde près de 450 kilomères d'anciens chemins forestiers, est une des constituantes de l'héritagequi interpelle levisiteur s'aventurant dansce territoire. Si l'on remarque encore les traces des coupes de bois, la nature reprend peu à peu sa place, un peu plus de dix ans après la reconnaissance formelle de ses droits. La lente récupération du milieu mènera à la reconstitution des peuplements d'origine représentatifs des Laurentides.

Sillonnant le fond des vallées, les routes du parcontlargementinfluencéle développement du territoire. Leschantiers, les camps forestiers, les écurieset la cuisine du «coolos se sont agrippés aux voies de pénétration. Aujourd'hui encore, les infrastructures d'accueil, les campings et les sentiers de randonnée sont situés là où les bûcherons avaient choisi de s'installer.

\section{De la chasse-galerie au canot-camping}

Les voies de pénétration ont non seulement influencé le développement du parc, mais elles ont également permis l'émergence de la pratique d'activités récréatives, Ainsi, la pêche a été, pendant longtemps, un attrait majeur dans le parc. Il va de soi que ce pays aux multiples lacs était appelé tout naturellement à offrir cette activité. Celle-ci a influencé de façon notable la gestion et le développement du territoire, Le résenu routier secondaire légué par l'industrie forestière s'est maintenu, avec les années, a fin de répondre aux besoins des pêcheurs.

Devant la popularité grandissante des loisirs de plein air, le parc s'est ajusté. La peche ne rejoint maintenant que $2 \%$ de la clientèle du parc. L'un des défis à relever est de rationaliser cette activité en favorisantl'acoès auxlacs quioffient lesmeilleurs potentiels, tout en réduisant le résenu routier menant aux lacs dont l'offre est plus marginale. Il s'agit, encore là, d'une façon pour le parc de réaffirmer si mission de conservation des ressources naturelles. L'arrière-pays reprendra ainsi ses caracté- ristiques de région sauvage et offrira au visiteur une expérience vraiment dépaysante.

Lorsqu’à la fin des années 1950 , le parc fut ouvert aux visiteurs, le camping a été l'une des activités qui a connu le plus de succès. Il s'est développé d'abord dans les secteurs déboisés délaissés par les bûcherons et l'aménagement des sites s'est amorcé sans normes rigoureuses. Il faudra plus de dix ans avant que des installations répondant à la fois aux besoins des visiteurs ainsi qu'aux principes de conservation ne voient lejour. On compte aujourd'hui plus de $1000 \mathrm{em}-$ placements de camping répartis sur l'ensemble du territoire. Après plus de 30 ans d'utilisation, les sites n'ont pas tous bien supporté l'usure du temps. Très utilisés par une clientèle grandissante, plusieurs montrent des signes de dégradation, affectant par le fait même la qualité de l'expérience queles campeurs recherchent dans un parc.

Devant cette situation, la gestion de cette forme d'hébergement, que plusieurs considèrent comme une activité en soi, représente maintenant, elle aussi, un défi important. Le parc cherche actuellement à concilier de façon optimale l'offre et la demande. Par la suite, il faudra résolument accentuer la restauration des sites dégradés en fonction de la capacité de support du parc. Enfin, de concert avec les représentants du milieu, le ministère de l'Environnement et de la Faune devra rechercher un meilleur équilibre entre l'offre d'hébergement en périphérie du parc et celle située à l'intérieur des limites du territoire. À cet égard, le pare suivra les tendances observées dans la plupart des parcs naturels de l'Amérique du Nord.

Témoin privilégié de la lente évolution des attitudesqu'ontaffichéeles Québécois face à la conservation du milieu naturel, Tremblant, le patriarche des parcs québécois, s'est lui aussi adapté, au fil du temps, a fin de mieux répondre aux attentes de ses visiteurs. Fréquenté d'abord par les inconditionnels de la grande nature quelques centaines de campeurs alors considérés comme marginaux - cette clientèle a rapidement été rejointe par des milliers de citadins à la recherche d'un havre de paix et de verdure. En 1971, on estimait la fréquentation du parc à près de 200000 visiteurs. En 1994, c'est près d'un million de personnes qui ont pratiqué une activitéà l'intérieur deslimites du territoire. 
De simple destination de camping, le parc s'est graduellement transformé en unevaste étendue de nature propice aux activités de plein air.

Pour répondre aux nouvelles tendances, le Ministère a consenti de nombreux efforts, dès le début des années 1970, afin de créer un réseau de sentiers de randonnée à skis, des circuits de canot-camping et de nombreux sentiers de randonnée pédestre qui donnent accès à des points de vue spectaculaires sur la vallée de la Diableet sur celle de la Pimbina. Avec l'aménagement d'un sentier de longue randonnée, fin 1970, le parç, petit à petit, laissait découvrir ses plus beaux atouts.

Une autre facette du parc lui confere un cachet distinctif. Le territoire est la source de trois bassins hydrographiques importants. Au centre du parc, les grands lacs donnent naissance à la rivière du Diablequi filevers la rivière Rouge. Cette dernière sé jettedans la rivière des Outaouais, quelques kilomètres en aval de Carillon. Tout à fait à l'est du parc, le lac de L'Assomption fait naître la rivière du même nom, qui atteint le fleuve Saint-Laurent en aval de l'île de Montréal. Enfin, la rivière Matawin prend sa source au nord du pare et alimente la rivièreSaint-Maurice. Cette extraordinaire richesse hydrographique assure aux résidants de la grande région de Montréal une source d'approvisionnement en eau dont les origines sont à l'abri de toute altération. Cette caractéristique constitue un attrait indéniable pour les canoteurs qui viennent pagayer sur les lacs et les rivieres de l'arrière-pays. Les circuits, conçus au cours des années 70 , attirent des centaines d'amateurs chaque année.

Le parc du Mont-Tremblant est un territoire résolument destiné à la protection et a la mise en valeur du milieu naturel. Les activités récréatives visent à mettre le visiteur en contact avec les aspects les plus saisissants de la nature sauvage. Alors que les aménagements destinés à la pratique des activités répondaient adéquatement au niveau de fréquentation des années 1970 , certains d'entre eux montrent des signes évidentsde détérioration tandis qued'autres font actuellement l'objet d'un contrôle étroit. Avec la popularité grandissante de certaines activités, telles que le vélo de montagne, il devient nécessaire de rewoir lesaménagementsafinde protéger la qualité du milieu naturel. L'expérience démontre, en effet, qu'une pratique d'activités res- pectueuse du milieu doit nécessairement être précédée par des aménagements adéquats. Les investissements de base sont alors largement compensés par la qualité de l'expérience que vit l'usager de même que par des niveaux d'entretien moins intenses.

\section{De la vision de "Jackrabbit" à la Station Mont-Tremblant}

Bien que les installations d'hébergementse trouvent en périphérie, l'activité ski est majoritairement située à l'intérieur des limites du parc. Les intrépides skieurs des années 1930 ont non seulement façonné la physionomie de cette partie du parc, mais ils ont été en quelque sorte les précurseurs de son visage actuel. On peut imaginer avec quelle fascination Johannsen a gravi les quelque 1000 mètres de ce massif et avec quel enthousiasme Joe Ryan a donné naissance à la station, la plus grande dans l'est du Canada.

Pour plusieurs, il n'existe pas de cohabitation possible entre le développement d'une station de ski et la notion de parc naturel. Il est vrai qu'au fil des années, le développement de la station de ski et celui des autres activités du parc se sont déroulés en parallèle. Cette dualité avait été exprimée lors des audiences publiques qui ont précédé la reconnaissanceofficielle du parc. Les participantsavaient alors opté majoritairement pour le maintien de la station à l'intérieur des limites du parc.

Depuis ce temps, des efforts de plus en plus importants sont consentis dans le but d'associer le développement d'activités récréatives à la protection et à la mise en valeur du milieu naturel, démontrant ainsi que des intérêts à prenière vue divergents peuvent se rejoindre. D'ailleurs des actions en partenariat sont actuellement en cours. Le savoir-faire du ministère de l'Environnement et de la Faune, dans sa mission de conservation et d'éduction, se marie à celui de la Station Mont-Tremblant, dans le but de mettre en valeur la montagne pour le bénéfice des urilisateurs. N'est-ce pas la l'accomplissement de la vision du legendaire sJackrabbiss?

\section{La noble vocation éducative}

Anjourd'hui, il semble tout naturel d'associer le parc du Mont-Tremblant à une mission éducative. Les forts courants mondiaux en faveur de l'environnement, initiés par la stratégie mondiale de conservation, le rapport Brundtland traitant des grandes próoccupationsenvironnementales et, tout dernièrement, la conférence de Rio, qui a donné naissance à la convention sur la biodiversité, ont largement sensibilisél'opinion publique au rổle fondamental que doivent jouer les espaces naturels et les parcs dans l'éducation à l'environnement.

Pour les visiteurs du pare du MontTremblant à l'approche des années 1970 , cettevocation était cependant nouvelle. Ils furent les premiers à expérimenter des activités d'interprétation de la nature dans les parcs québécois. Des naturalistes rassemblaient alors adultes et enfants pour leur faire découvrir la vie du castor ou encore, pour dresser un inventaire de crapauds etdegrenouillesafin d'en saisir toute la richesse écologique.

De nos jours, la conscience environnementale s'est développée au sein de la population. La notion d'interprétation de la nature a elle aussi évolué. Toujours aussi populaires, les activités d'interprétation tendent maintenant à faire découvrir les attraits de la nature tout en mettant en valeur le rôle des pares dans la préservation du milieu naturel.

L'éducation au milieu naturel peut prendre toutes sortes de formes : un sentier de découverte de la nature bien aménagé; une piste de vélo de montagne en restauration; un naturaliste qui accompagne un groupe de visiteurs; un gardien de territoire qui intervient auprès d'usagersen encourageant l'adoption de comportements respectueux du milieu naturel.

\section{L'avenir: la nature en héritage}

Le périple du centenaire se poursuit en faisant un bond de quelques décennies, transportant le visiteur dans l'avenir, en l'an 2050! Les limites de la ville de Montréal s'étendent jusqu’à Saint-Jérồme. Du haut d'une tour à bureau, à la sortie du métro Sainte-Adele, on voit se profiler au loin l'undes derniersespacesverts préservés. Pure spéculation? Qui sait quelle sera l'ampleur du développement au cours du prochain siècle? La société réussira-t-elle à concilier l'essor économique et la préservation de la diversité biologique? 
Forcés de composer avec leur popularité croissante, les parcs concentrent leurs efforts à bien maîtriser leurs mandats de récréation et de conservation, même s'ils peuvent sembler, à certains égards, inconciliables. Les préoccupations de préservation des ressources se heurtent en effet, bien souvent, aux besoins toujours plus grands de la population qui sćjourne en milieu naturel. Les demandes sont légitimes pourtant, car lesparcs nesa uraient exister sins que leurs propriétaires, les citoyens, n'y aient accès. Le défi est réel: une fréquentation démesurée ou une offre d'activités non respectueuses du milieu naturel risquent d'annihiler, de manière irréversible, les efforts de conservation consentis au fil des ans, efforts qui traduisent l'essence même de ces territoires.

L'avenir du pare du Mont-Tremblant trouve donc sa place dans un juste équilibre entre la conservation du patrimoine naturel et sa mise en valeur pour les visiteurs. Conscient du dilemme, le parc continue d'investir dans l'acquisition de connaissances en vue de protéger et de restaurer le milieu naturel. La connaissance des éoosystèmes et de leurs réactions à la présence humaine est effectivement indispensable pour en assurer le maintien. De même, 'éducation au milieu naturel s'avère un outil privilégié pour atteindre les mandats dévolus au parc. De plus sa vocation récréative est indéniable; les activités et services actuels ainsi que les nouveaux produits offerts doivent contribuer à promouvoir l'in tégrité et la beauté des paysages laurentiens. Lavenir du parc réside dans cette volonté d'agencer finement les préoccupationsenmatèredeconservation, d'éducation et de récréation.

\section{Un territoire en évolution}

Aujourd'hui encore, le parc du MontTremblant témoigne de l'héritage légué par ses pères. De la légende de Maniounga Soutana aux chantiers des vaillants bûcherons, le milieu porte encore quelques cicatrices des formes traditionnelles d'utilisation du territoire. $A$ l'époque, on ne percevait pas l'exploitation des ressources naturelles comme une menace possible à leur conservation. Cette utilisation ancestrale du milieu répondait aux aspirations de la société québécoise d'alors.

Malgré son grand âge, la parc a vécu une véritable renaissance lors de sa reconnais- sance officielle en 1981. Depuis, ils simprègne progressivement des caractéristiques inhérentes aux grandls espaces protégés à travers le monde. Cette transformation doit se dérouler dans le respect de la triple vocation du parcet desa personnalité. Sans renier ses origines, le territoire fait maintenant l'objet d'une protection intégrale contre l'exploitation des ressources. Les cicatrices se referment et laissent émerger, avec toute leur majesté, les spectaculaires paysiges laurentiens.

Témoin de la lente évolution des attitudes qu'ont affichéles Québécois face au milieu nature, le parc du Mont-Tremblant est maintenant résolument destiné à la protection et à la mise en valeur du milieu, tout en assurant aux citoyens des expériences récréntives de qualité. Le patriarche entreprend, avec sagesse, son deuxième siède, confiant que les générations futures continueront à bénéficier de cette vaste contrée où le voyageur peut, à loisir, découvrir sa vraie nature.

\section{REEFÉRENCES}

BOUROAGES, Jean-Luc, et al, Les parcs naturels du Canada et du Ouebec, Pollitiques, lois et roglaments, Urwersité de Mantréal, 1994, $125 \mathrm{p}$.

FOURNIER, Marcel, Histoire du parc du Mont-Tromblant. Des originas a 1981, Montréal, ministere du Loisir, de la Chasse et de la Pêche, 1981,91 p.

LAUFIN, Serge. Histoire des Laurentides. Colection Les régions du Quebec 3. Québec, Institut québécois de recherche sur la culture, $1989,892 \mathrm{p}$

LIPPE, Loulse, Etude des possibilités d'aménagement touristique du parc du Mont-Tremblant. Thèse, Universitè de Montréal, 1961, 126 p.

MONGEAU, Jean-Fene, Le Quebec pord l'un de ses plus eminenis biologistes, dans In vivo, bulletin del'Association des biologistes du Québec, vol. 10 , no 2, marsavil 1990, pp. 12-21. 